\title{
PREPARATION AND EVALUATION OF SURFACE MODIFIED NANOPARTICLES OF CALCIUM PHOSPHATE AS EXTRACT CARRIER
}

\author{
SRITOMA BANERJEE ${ }^{*}$, KALYAN K. SEN ${ }^{1}$
}

\author{
1Department of Pharmaceutical Technology, Gupta College of Technological Sciences, Ashram More, G. T. Road, Asansol 713301, West \\ Bengal, India \\ Email: sritomaaa@gmail.com
}

Received: 02 May 2020, Revised and Accepted: 09 Jun 2020

\begin{abstract}
Objective: The aim of this study was to develop, optimize and characterize carbohydrate coated calcium phosphate nanoparticles of Chelidonium majus $L$. extract along with carried out in vivo study to observe activity in the liver.

Methods: Surface modified calcium phosphate nanoparticles of Chelidonium majus L. extract were developed and optimized. Extract loading and particle size were the two responses, effects on which were analyzed. Characterization studies, in vitro extract release and in vivo distribution studies were carried out. Also in vivo histopathological analysis was carried out to observe effects of extract loaded nanoparticles in liver of wistar albino rats in paracetamol, rifampicin-isoniazid, cisplatin and carbon tetrachloride-induced hepatotoxicity.

Results: Pareto chart and surface response curve indicated that sonication time, the concentration of lactose and concentration of extract were important factors affecting particle size and extract loading. ANOVA was performed and obtained data pointed out that model was significant for both responses. Particle size and zeta potential results indicated the stability of prepared nanoparticles along with extract was loaded (37.22\%) satisfactorily on coated cores. Characterization studies indicated no interaction between the components and also extract release demonstrated diffusion-controlled mechanism. These extract loaded nanoparticles were largely found in the liver than heart, lungs. Hepatoprotective activity of nanoparticles of the extract was confirmed by correlating histopathology results of normal, toxic, silymarin treated, extract-treated and formulation treated groups.
\end{abstract}

Conclusion: Lactose coated nanoparticles of calcium phosphate proved to be excellent carriers of plant extract. These nanoparticles efficiently targeted liver and generated cellular protective action in hepatic damage.

Keywords: Calcium phosphate, Surface modification, Statistical optimization, Chelidonium majus L., Distribution, Hepatoprotective

(C) 2020 The Authors. Published by Innovare Academic Sciences Pvt Ltd. This is an open access article under the CC BY license (http://creativecommons.org/licenses/by/4.0/) DOI: http://dx.doi.org/10.22159/ijap.2020v12i4.38126. Journal homepage: https://innovareacademics.in/journals/index.php/ijap

\section{INTRODUCTION}

A nanoparticulate drug delivery system is an exclusive area of drug delivery which has significant diversity in its own system. Each has its own pertinent attributes and exertions. Most significantly, this domain of drug delivery system is expanding day by day with one type trying to overcome the limitations of the previous type. The principal advantage of nanoparticulate drug delivery is related to the vast surface area exposure due to the smaller particle size. Aquasome is one of those newly developed drug delivery system in this area. It is a self-assembled and three-layered surface-modified nanoparticulate system which was invented by Nir Kossovsky in 1994 [1]. There are three layers in the system with each have its own crucial role to the entire system. These are core, coating layer of polyhydroxy oligomer and layer of the attached biochemically active molecule. Core is generally composed of nanocrystalline tin oxide, carbon, calcium phosphate and hydroxyapatite. Calcium phosphate and hydroxyapatite are the most preferred cores of choice. Bioceramics, mainly calcium phosphate is used in bone grafting, tissue engineering because it is the principal component of bone. Due to its natural occurrence in the human body, it is biocompatible and biodegradable in nature. Surface modification was done by coating ceramic core with polyhydroxy oligomer or carbohydrates. Carbohydrates provide a natural stabilizing effect to the biochemically active molecule as well as the whole drug delivery system. It provides an aqueous like environment to preserve the stability of the biological molecule. So, the probability of toxicity generated by the instability of drug delivery system itself can be irradiated. These surface-modified nanoparticles proved to be efficient enough for successful delivery of haemoglobin [2, 3], insulin [4], antigen [5-7], poorly aqueous soluble drugs [8-10], enzyme [11], vaccine [12], human interferon [13].

Chelidonium majus L. (Family-Papaveraceae), also commonly known as greater celandine is a well known medicinal plant indigenous to Europe and Asia. A plant rich with isoquinoline alkaloids also contains divergent class of components [14] like phenolics, flavonoids, flavonoidal glycosides, saponins, sterols, organic acids, amino acids. Its empirical application as folk medicine made its way throughout the vast topography of Europe and also in China. Principal and noteworthy applications of Chelidonium majus L., were involved in treatment of skin diseases, eye infections, and liver disorders in traditional herbal medicinal system and in homeopathy [15].

In this study, aquasome based on calcium phosphate was developed, surface modified and loaded with Chelidonium majus $L$. extract (henceforth it will be called as extract), as a drug delivery system. Formulations were developed by statistical design with variation of different essential factors during preparation and optimized accordingly. After optimization, selected formulation had undergone a characterization study along with in vitro extract release process. Also distribution of these extract carriers was observed in liver, lungs and heart of wistar albino rats. Prepared aquasomes of extract was administered to wistar albino rats to observe protective effects and nature of protection in the liver. It can be stated that these surface-modified extract carriers of calcium phosphate nanoparticles were very much biological or natural due to presence of biological components into it.

\section{MATERIALS AND METHODS}

\section{Materials}

Chelidonium majus L. was purchased as a whole dried herb from Associated Traders, Kolkata, India and due to this authentication process was done by thin-layer chromatography according to French Pharmacopoeia, 2002. Details of the procedure were mentioned in our previous research paper [16]. Acetone and disodium hydrogen phosphate were purchased from Merck Specialities Private Limited (Mumbai, India). Calcium chloride was purchased from Qualigens Fine Chemicals (Mumbai, India). Lactose monohydrate was purchased from Loba Chemie Private Limited (Mumbai, India). 
Silymarin, carbon tetrachloride, fluorescein isothiocyanate-dextran and 0.2 micron membrane filter paper were purchased from SigmaAldrich (Bangalore, India). Formaldehyde was purchased from Thermo Fisher Scientific India Private Limited (Mumbai, India). Rifampicin and isoniazid were obtained as a gift sample from Lupin Limited, India. Paracetamol was obtained also as gift sample from Cipla Limited, India). Cisplatin injection was purchased (KEMOPLAT, Fresenius Kabi Oncology Limited, India). All other reagents were of analytical grade. Protocol of in vivo experiment was approved by the Institutional Animal Ethical Committee of Gupta College of Technological Sciences, Asansol, West Bengal (protocol no. GCTS/IAEC/2016-March/09).

\section{Preparation of plant extract}

Extract was prepared by using the method described in our previous research paper [16].

\section{Preparation of aquasomes of extract}

Aquasomes were prepared using the method of Vengala et al., 2013 [10], with required modifications. A statistical design method was utilized using Design-Expert software (Version 12.0.2.0, Trial version by Stat-Ease, Inc., Minneapolis, USA) to prepare and optimize different formulations by varying different parameters which were illustrated in table 1. Here Plackett Burman design was followed with eleven factors, including one dummy factor. Factors were sonication time (A), the concentration of calcium chloride (B), sonication temperature $(C)$, the concentration of lactose (D), amount of acetone (E), amount of core (F), amount of drug/extract (G), time of contact during drug/extract incubation $(\mathrm{H})$, the temperature of drug/extract incubation (J), RPM of stirrer at drug/extract incubation (K) and dummy (L). Total number of formulation developed was 12 .

\section{Preparation included three steps}

\section{Core preparation}

Disodium hydrogen phosphate solution was added slowly to calcium chloride solution under sonication (Probe Sonicator, Frontline Electronics and Machinery Private Limited, Gujarat, India). Formed precipitate was separated by centrifugation (Cooling Centrifuge, Remi Elektrotechnik Limited, Maharashtra, India) and washed with double distilled water. Then it was suspended in double distilled water to filter through 0.2 micron membrane filter and filtered volume was freeze-dried. In this study, cores were prepared by varying concentrations of calcium chloride (mol) and the duration of sonication ( $\mathrm{min})$ at different temperature $\left({ }^{\circ} \mathrm{C}\right)$ as depicted in table 1 .

Table 1: Formulation optimization-1

\begin{tabular}{|c|c|c|c|c|c|c|c|c|c|c|c|}
\hline Formulation code & $\mathbf{A}$ & B & $\mathbf{C}$ & D & $\mathbf{E}$ & $\mathbf{F}$ & $\mathbf{G}$ & $\mathbf{H}$ & $\mathbf{J}$ & $\mathbf{K}$ & $\mathbf{L}$ \\
\hline F1 & 150 & 0.375 & 4 & 0.2 & 1 & 500 & 0.5 & 6 & 6 & 1 & -1 \\
\hline $\mathrm{F} 2$ & 30 & 0.375 & 30 & 0.04 & 1 & 500 & 2.5 & 6 & 6 & -1 & 1 \\
\hline F3 & 150 & 0.25 & 30 & 0.2 & 0 & 500 & 2.5 & 24 & 6 & -1 & -1 \\
\hline F4 & 30 & 0.375 & 4 & 0.2 & 1 & 50 & 2.5 & 24 & 30 & -1 & -1 \\
\hline F5 & 30 & 0.25 & 30 & 0.04 & 1 & 500 & 0.5 & 24 & 30 & 1 & -1 \\
\hline F6 & 30 & 0.25 & 4 & 0.2 & 0 & 500 & 2.5 & 6 & 30 & 1 & 1 \\
\hline F7 & 150 & 0.25 & 4 & 0.04 & 1 & 50 & 2.5 & 24 & 6 & 1 & 1 \\
\hline F8 & 150 & 0.375 & 4 & 0.04 & 0 & 500 & 0.5 & 24 & 30 & -1 & 1 \\
\hline F9 & 150 & 0.375 & 30 & 0.04 & 0 & 50 & 2.5 & 6 & 30 & 1 & -1 \\
\hline F10 & 30 & 0.375 & 30 & 0.2 & 0 & 50 & 0.5 & 24 & 6 & 1 & 1 \\
\hline F11 & 150 & 0.25 & 30 & 0.2 & 1 & 50 & 0.5 & 6 & 30 & -1 & 1 \\
\hline F12 & 30 & 0.25 & 4 & 0.04 & 0 & 50 & 0.5 & 6 & 6 & -1 & -1 \\
\hline
\end{tabular}

\section{Polyhydroxy oligomer coating}

Suitable amount of dried ceramic core was added to the lactose solution in double-distilled water and sonicated. It was shaken in a shaker incubator bath and acetone was added after that. The dispersion was centrifuged and coated cores were collected by decanting off the supernatant liquid and washing with double distilled water. Coated cores were dried at $70{ }^{\circ} \mathrm{C}$ in a hot air oven. In this step, carbohydrate concentration (mol), amount of core (mg) and amount of acetone $(\mathrm{ml})$ were varied (table 1$)$.

\section{Extract loading}

Extract was added to aqueous dispersion of coated core. It was shaken in a shaker incubator bath for suitable period of time and after that the dispersion was centrifuged. Extract loaded aquasomes were collected by decanting off the supernatant liquid and air dried. Concentration (\% W/V) was varied along with stirring speed (RPM), incubation period $(\mathrm{h})$ and temperature $\left({ }^{\circ} \mathrm{C}\right)$ in different formulations as depicted in table 1 to determine suitable amount of extract loading. Further variations were done by changing concentration of extract and time of stirring to obtain optimum and suitable extract loading.

\section{Quantification of extract loading in aquasomes}

During the extract loading process, dispersion was centrifuged after shaking for a suitable period of time. After centrifugation supernatant liquid was collected and absorbance of this supernatant was measured at $272 \mathrm{~nm}$ using UV-visible spectroscopy (Thermo Spectronic UV1 UV-Visible Spectrophotometer, USA). Wavelength was selected as per the result of previous research work by the same author [17]. Percentage extract loading was calculated by comparing it with blank.

\section{Calcium alginate coating}

Calcium alginate coating was done on extract loaded nanoparticles for oral administration [11] during in vivo study. Aquasomes were dispersed in sodium alginate solution $(0.1 \% \mathrm{w} / \mathrm{v})$ and added dropwise to calcium chloride solution $(1 \% \mathrm{w} / \mathrm{v})$. After $20 \mathrm{~min}$ of the incubation period, particles were filtered, washed and dried at room temperature. Amount of nanoparticles entrapped in calcium alginate was determined with the help of the amount of remaining portion of nanoparticles left in sodium alginate dispersion.

\section{Particle size and zeta potential determination}

Particle size and zeta potential were measured (Malvern Zetasizer Nano ZS, United Kingdom) to assess the size and stability of prepared nanoparticles (without alginate coating). Before particle size and zeta potential measurement, samples were dispersed in double distilled water and sonicated for $5 \mathrm{~min}$. For zeta potential measurement, sonicated formulations were taken into zeta dip cell. All the measurements were done at $25^{\circ} \mathrm{C}$.

\section{Fourier transform infrared spectroscopy (FTIR)}

FTIR spectra of Chelidonium majus L. extract and aquasomes loaded with extract were recorded using FTIR spectrophotometer (Shimadzu IR-Prestige 21, Shimadzu Scientific Corporations Inc., USA). Solid sample was mixed with $\mathrm{KBr}$ in a ratio of $1: 100$ and grounded thoroughly. This mixture was pressed with the use of a hydraulic press to form a pellet. This pellet was placed in the sample holder. Liquid sample was placed in liquid cell. Spectra were recorded in the wavelength region of $400-4000 \mathrm{~cm}^{-1}$.

\section{Differential scanning calorimetry (DSC)}

In this study, core, coated core and extract loaded formulations were undergone thermal analysis with the help of a differential scanning 
calorimeter (Model-Q10, TA Instruments, USA). Samples were heated from $25^{\circ} \mathrm{C}-300^{\circ} \mathrm{C}\left(10{ }^{\circ} \mathrm{C} / \mathrm{min}\right)$.

\section{X-ray diffraction study (XRD)}

XRD study was performed to analyze the crystalline behavior of extract loaded nanoparticles using X-ray diffractometer (SmartLab, Rigaku Corporation, Japan) within the range of $0{ }^{\circ} \mathrm{C}-80^{\circ} \mathrm{C}$.

\section{In vitro dissolution study}

In vitro dissolution study was carried out to understand extract release from formulation over a period of time. Here it was carried out by dialysis method $[17,18]$ in $\mathrm{pH} 6.8$ phosphate buffer. Nanoparticles (without alginate coating) containing $100 \mathrm{mg}$ of extract was taken in a dialysis bag. This enclosed dialysis bag was kept in $200 \mathrm{ml}$ of $\mathrm{pH} 6.8$ phosphate buffer medium and entire medium was under motion with the help of magnetic stirring at 37 ${ }^{\circ} \mathrm{C}$. At a definite time interval, a specific amount of sample was withdrawn with the replacement of a similar amount buffer. Release was checked after the first $30 \mathrm{~min}$ and then at $1 \mathrm{~h}$ interval up to $8 \mathrm{~h}$. Then, after $24 \mathrm{~h}$, the sample was withdrawn to observe any extract release. Absorbance of each sample was determined in UV spectrophotometer at $272 \mathrm{~nm}$. This UV method was validated analytically. A detail of method development and validation was mentioned in our previous research paper [16].

\section{Preparation of fluorescent doped aquasomes of Chelidonium majus L. extract}

Fluorescein isothiocyanate-dextran was loaded in calcium phosphate cores of aqua some. Fluorescent doped cores were prepared by considering the procedure by Roy et al., 2014 [19]. Here procedure followed was the same as F7 core preparation. Only fluorescein isothiocyanate-dextran $(10 \mathrm{mg} / \mathrm{ml})$ was dissolved in a disodium hydrogen phosphate solution. Prepared extract loaded nanoparticles were entrapped in alginate.

\section{Distribution of fluorescent doped aquasomes in different organs of rats}

This study was carried out to observe aquasomes distribution in different organs like the liver, heart and lungs of albino wistar rats. Fluorescein isothiocyanate-dextran doped alginate coated aquasomes (containing $500 \mathrm{mg} / \mathrm{kg}$ body weight of extract) were orally fed to wistar albino rats. After $24 \mathrm{~h}$, rats were sacrificed.
Specimens of liver, lung and heart were collected for histopathology. These histopathology slides were observed under a fluorescence microscope (Leica DM 1000, Leica Microsystems, Mumbai, India).

\section{In vivo study}

Healthy wistar albino rats (bodyweight of 150-200 g) of both sexes were selected randomly and kept in their respective cages fifteen days before beginning the study. Animals were kept in a $12 \mathrm{~h}$ light and dark environment each day with food and water ad libitum. Temperature and relative humidity in the animal keeping area was maintained as $22^{\circ} \mathrm{C}\left( \pm 3^{\circ} \mathrm{C}\right)$ and $50-60 \%$.

Each group consisted of six rats selected randomly. Details of administered dose were given in table 2. Total duration of dosing was for fifteen days. Normal control group (Gr-N) was orally administered with normal saline daily. Paracetamol induced hepatotoxic group (Gr-P1) was administered with paracetamol as a single dose on $15^{\text {th }} \mathrm{d}$ orally. Silymarin treated group (Gr-P2) was administered with silymarin daily and paracetamol the same as GrP1. CMF (Gr-P3) and CME (Gr-P4) treated groups were administered with CMF, CME, orally daily and paracetamol the same as Gr-P1 to each. Rifampicin-isoniazid induced hepatotoxic group (Gr-RI1) was administered each rifampicin and isoniazid daily through the oral route. Silymarin treated group (Gr-RI2) was administered with silymarin daily and rifampicin with isoniazid the same as Gr-RI1. CMF (Gr-RI3) and CME (Gr-RI4) treated groups were administered with CMF, CME, orally daily and rifampicin with isoniazid same as Gr-RI1 to each. Cisplatin-induced hepatotoxic group (Gr-C1) was administered with cisplatin intraperitoneal (i. p.) as a single dose on $15^{\text {th }} \mathrm{d}$. Silymarin treated group (Gr-C2) was treated with silymarin orally daily and cisplatin the same as Gr-C2. CMF (Gr-C3) and CME (Gr-C4) treated groups were administered with CMF, CME, orally daily and cisplatin the same as Gr-C2 to each.

Normal control CIN group (Gr-ClN) was administered with normal saline orally daily and vehicle without carbon tetrachloride i. p. twice in a w. $\mathrm{CCl}_{4}$ was administered as a single dose to induce hepatotoxicity twice in a w (Gr-Cl1). Silymarin treated group (Gr-Cl2) was administered with silymarin orally daily and $\mathrm{CCl}_{4}$ same as Gr-Cl1. CMF (Gr-Cl3) and CME (Gr-Cl4) treated groups were administered with $\mathrm{CMF}, \mathrm{CME}$ orally daily and $\mathrm{CCl}_{4}$ same as Gr-Cl1 to each.

On $16^{\text {th }} \mathrm{d}$ rats were sacrificed and liver specimens were collected for histopathological analysis from respective groups.

Table 2: Details of administered dose during in vivo study

\begin{tabular}{ll}
\hline Description of administration & Dose \\
\hline Alginate coated Chelidonium majus L. extract loaded nanoparticles & amount containing $500 \mathrm{mg} / \mathrm{kg}$ bodyweight of extract \\
Chelidonium majus L. extract & $500 \mathrm{mg} / \mathrm{kg}$ bodyweight of extract \\
Silymarin & $200 \mathrm{mg} / \mathrm{kg}$ bodyweight \\
Paracetamol & $2 \mathrm{~g} / \mathrm{kg}$ bodyweight \\
Rifampicin-isoniazid & Each $100 \mathrm{mg} / \mathrm{kg}$ bodyweight \\
Cisplatin & $7.5 \mathrm{mg} / \mathrm{kg}$ bodyweight \\
Carbon tetrachloride & $30 \%$ solution in liquid paraffin, $1 \mathrm{ml} / \mathrm{kg}$ body weight \\
\hline
\end{tabular}

\section{RESULTS AND DISCUSSION}

\section{Formulation development and optimization}

Nanoparticles preparation involved three steps. In each step there were various critical factors. In core preparation step, sonication temperature and duration of sonication were important factors which can cause serious effect on particle size, surface activity and subsequent carbohydrate adsorption. Whereas in the coating stage, amount of core, the concentration of carbohydrate and amount of acetone were the most important factors which also can generate variable results in particle size and further extract loading. During extract adsorption, the principal concern was with amount of extract adsorbed. This effect could be influenced by factors like amount of extract, stirring speed, the temperature of incubation and duration of stirring or incubation. Since aquasome preparation involves the association of multiple factors, it is logical to use a statistical design to optimize the formulation development process. Using PlackettBurman design helped to eradicate unintended factors. By selecting high and low range of each variable, it was suitable and efficient to stigmatize how a specific response was altered or rather precise to say which factors were important in a specific step of a particular experiment. Two responses were analyzed in this design. Response 1 was drug/extract loading and Response 2 was particle size.

It was found that extract was loaded with satisfactory percentage loading in each formulation. All the extract loaded aquasomes exhibited good particle size (less than $300 \mathrm{~nm}$ ) as shown in table 3. Zeta potential values indicated the stability of prepared formulations. Also all the formulations except F-3, F-9 and F-11 exhibited good zeta potential values as shown in table 3. Low zeta potential values of F-3, F-9 and F-11 might be due to a slight increase in agglomeration behaviour of calcium phosphate, which was also in supportive with the higher particle size of those formulations. 
Table 3: Extract loading quantification along with respective particle size and zeta potential data

\begin{tabular}{|c|c|c|c|}
\hline Formulation code & Extract loading (\%) & Particle size (nm) & Zeta potential (mV) \\
\hline F1 & $14.32 \pm 0.71$ & $165.00 \pm 2.29$ & $-21.15 \pm 0.11$ \\
\hline $\mathrm{F} 2$ & $08.66 \pm 0.28$ & $137.87 \pm 1.69$ & $-20.22 \pm 0.08$ \\
\hline F3 & $12.97 \pm 0.77$ & $268.95 \pm 0.51$ & $-14.17 \pm 0.17$ \\
\hline $\mathrm{F} 4$ & $16.85 \pm 1.66$ & $135.45 \pm 0.70$ & $-22.42 \pm 0.10$ \\
\hline F5 & $27.21 \pm 1.13$ & $125.80 \pm 2.43$ & $-20.77 \pm 0.10$ \\
\hline F6 & $25.32 \pm 0.87$ & $131.10 \pm 1.00$ & $-22.60 \pm 0.07$ \\
\hline F7 & $30.97 \pm 0.66$ & $114.72 \pm 0.75$ & $-23.52 \pm 0.10$ \\
\hline F8 & $17.43 \pm 0.95$ & $161.10 \pm 0.86$ & $-20.82 \pm 0.08$ \\
\hline F9 & $12.35 \pm 0.53$ & $214.37 \pm 0.68$ & $-19.55 \pm 0.11$ \\
\hline F10 & $22.10 \pm 1.28$ & $162.45 \pm 1.31$ & $-20.27 \pm 0.04$ \\
\hline F11 & $28.32 \pm 1.52$ & $281.37 \pm 0.71$ & $-13.77 \pm 0.10$ \\
\hline F12 & $17.10 \pm 0.56$ & $125.80 \pm 0.82$ & $-22.07 \pm 0.04$ \\
\hline
\end{tabular}

Each data represent the average of three independent experiment \pm SD

ANOVA was carried out to determine the significance of the fitted model. Model was significant both in case of extract loading and particle size. Table 4 showed model $\mathrm{F}$ value for drug/extract loading was 874.77 denoting model was significant for extract loading. Table 5 showed model $F$ value for particle size was 39.85 implying that the model was significant for particle size also. Factors generating $p$ values less than 0.0500 were significant model terms while $p$ values greater than 0.1000 implied that model terms were not significant. Speed of stirrer, time of contact and temperature during extract incubation (above t-value limit) were crucial factors during extract loading on coated calcium phosphate cores (fig. 1A). Sonication time, sonication temperature and concentration of lactose were significant model terms (above t-value limit) influencing particle size of extract loaded aquasomes (fig. 1B). Surface response image of particle size (fig. 2A) showed that particle size was descending with an increase in the concentration of calcium chloride while ascending with increase in sonication time. Surface response diagram of drug/extract loading (fig. 2B) indicated to the fact that low concentration of calcium chloride and higher sonication time individually increased extract loading. Concentration of lactose was an important factor in both cases, causing an increase in particle size and extract loading with increase in concentration. It was also found to be interesting that decrease in sonication temperature caused a decrease in particle size while an increase in extract loading. Also, particle size was decreased with increasing speed of stirrer. Final equations in terms of coded factors for extract loading and particle size were depicted in equation (1) and (2)

Extract loading $=+19.47-4.18 \mathrm{~B}-0.8650 \mathrm{C}+0.5133 \mathrm{D}+1.59 \mathrm{E}-1.81 \mathrm{~F}$ $1.61 \mathrm{G}+1.79 \mathrm{H}+1.78 \mathrm{~J}+2.58 \mathrm{~K}+2.67 \mathrm{~L}(1)$

Particle size $=+168.67+32.25 \mathrm{~A}-5.96 \mathrm{~B}+29.80 \mathrm{C}+22.06 \mathrm{D}-8.63 \mathrm{E}-7.25 \mathrm{H}+$ 6.20J-16.42K-3.90L (2)

Table 4: ANOVA for the selected factorial model (response 1: extract/drug loading)

\begin{tabular}{|c|c|c|c|c|c|c|}
\hline Source & Sum of squares & Degrees of freedom & Mean square & F-value & $p$ value & \\
\hline Model & 564.52 & 10 & 56.45 & 874.77 & 0.0263 & significant \\
\hline B-Calcium chloride & 209.84 & 1 & 209.84 & 3251.59 & 0.0112 & \\
\hline C-sonication temperature & 8.98 & 1 & 8.98 & 139.13 & 0.0538 & \\
\hline D-concentration of lactose & 3.16 & 1 & 3.16 & 49.00 & 0.0903 & \\
\hline E-amt of Acetone & 30.27 & 1 & 30.27 & 469.12 & 0.0294 & \\
\hline F-amt. of core & 39.53 & 1 & 39.53 & 612.56 & 0.0257 & \\
\hline G-amt of drug & 31.23 & 1 & 31.23 & 484.00 & 0.0289 & \\
\hline H-time of contact & 38.38 & 1 & 38.38 & 594.69 & 0.0261 & \\
\hline J-temperature of drug incubation & 38.02 & 1 & 38.02 & 589.17 & 0.0262 & \\
\hline K-rpm of stirrer at drug incubation & 79.77 & 1 & 79.77 & 1236.16 & 0.0181 & \\
\hline L-dummy2 & 85.33 & 1 & 85.33 & 1322.31 & 0.0175 & \\
\hline Residual & 0.0645 & 1 & 0.0645 & & & \\
\hline Cor Total & 564.59 & 11 & & & & \\
\hline
\end{tabular}

$p$ value $<0.0500$ significant model terms, $p$ value $>0.1000$ non-significant model terms

Table 5: ANOVA for the selected factorial model (response 2: particle size)

\begin{tabular}{|c|c|c|c|c|c|c|}
\hline Source & Sum of squares & Degrees of freedom & Mean square & F-value & $p$-value & \\
\hline Model & 34811.20 & 9 & 3867.91 & 39.85 & 0.0247 & significant \\
\hline A-sonication time & 12483.33 & 1 & 12483.33 & 128.62 & 0.0077 & \\
\hline B-Calcium chloride & 426.02 & 1 & 426.02 & 4.39 & 0.1712 & \\
\hline C-sonication temperature & 10658.86 & 1 & 10658.86 & 109.82 & 0.0090 & \\
\hline D-concentration of lactose & 5837.08 & 1 & 5837.08 & 60.14 & 0.0162 & \\
\hline E-amt of Acetone & 893.72 & 1 & 893.72 & 9.21 & 0.0936 & \\
\hline H-time of contact & 631.33 & 1 & 631.33 & 6.50 & 0.1254 & \\
\hline J-temperature of drug incubation & 461.28 & 1 & 461.28 & 4.75 & 0.1611 & \\
\hline K-rpm of stirrer at drug incubation & 3237.37 & 1 & 3237.37 & 33.36 & 0.0287 & \\
\hline L-dummy2 & 182.21 & 1 & 182.21 & 1.88 & 0.3042 & \\
\hline Residual & 194.11 & 2 & 97.05 & & & \\
\hline Cor Total & 35005.31 & 11 & & & & \\
\hline
\end{tabular}

$p$ value $<0.0500$ significant model terms, $p$ value $>0.1000$ non-significant model terms 


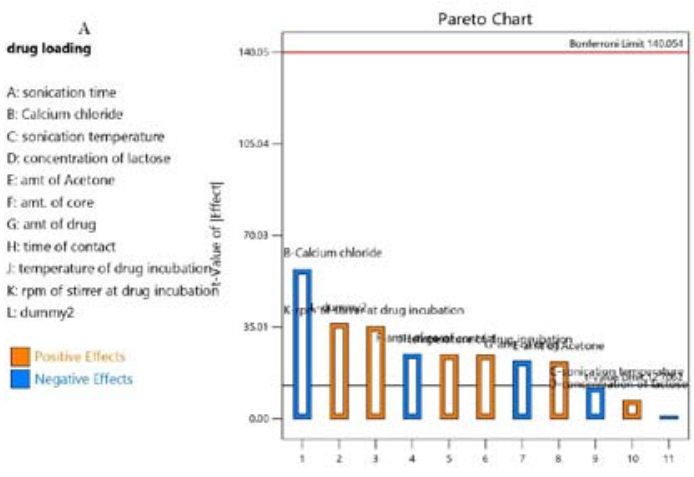

Rank
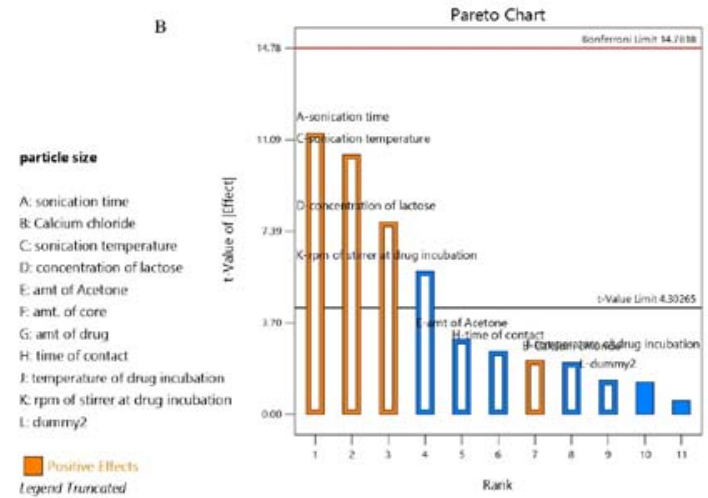

Rarik

Fig. 1: Pareto chart for extract/drug loading (A) and particle size (B)
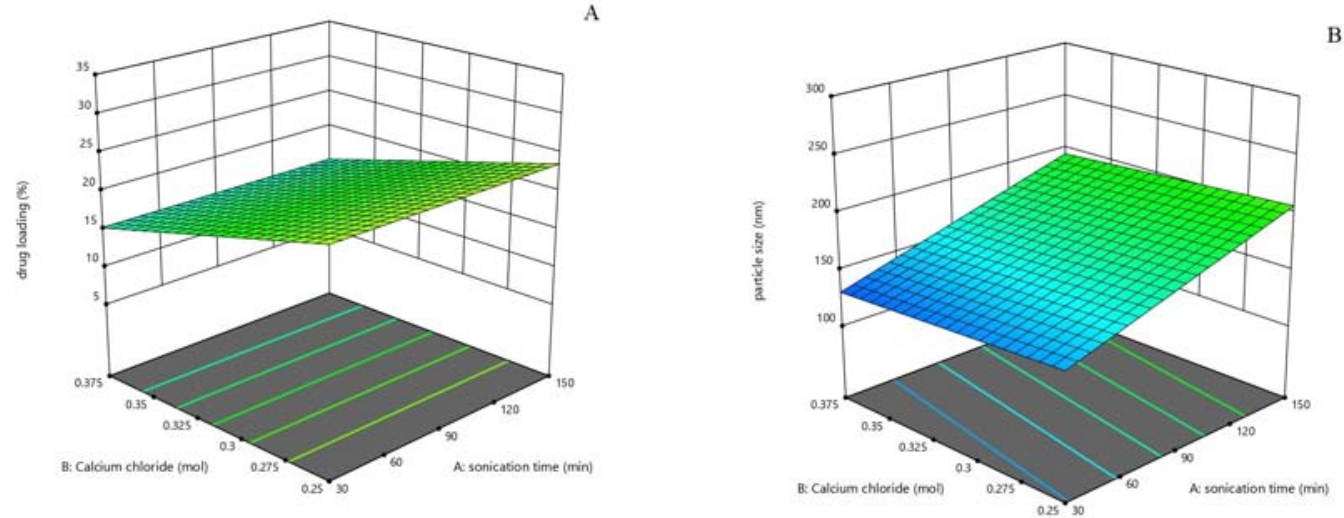

Fig. 2: Surface response diagrams for extract/drug loading (A) and particle size (B)

In fit statistics data (table 6), $\mathrm{R}^{2}$ value is the determination of the amount of variation around the mean value interpreted by model itself. It was observed that the predicted $\mathrm{R}^{2}$ value was in reasonable agreement with adjusted $\mathrm{R}^{2}$ value for both particle size and extract loading. In both of the case values were less than
0.2. Adequate precision is demonstrated to describe signal to noise ratio, the value of which greater than 4 is acceptable. It was observed in table 6 that for extract loading, adequate precision was 92.3310 and for particle size, it was 18.1773. Both values indicated a good signal.

Table 6: Fit statistics for extract loading and particle size

\begin{tabular}{|c|c|c|c|c|c|c|c|}
\hline \multicolumn{4}{|l|}{ Extract loading } & \multicolumn{4}{|l|}{ Particle size } \\
\hline Std. Dev. & 0.2540 & $\mathrm{R}^{2}$ & 0.9999 & Std. Dev. & 9.85 & $\mathrm{R}^{2}$ & 0.9945 \\
\hline Mean & 19.47 & Adjusted $\mathrm{R}^{2}$ & 0.9987 & Mean & 168.66 & Adjusted $\mathrm{R}^{2}$ & 0.9695 \\
\hline $\begin{array}{l}\text { Coefficient of } \\
\text { variation }\end{array}$ & 1.30 & Predicted $\mathrm{R}^{2}$ & 0.9835 & $\begin{array}{l}\text { Coefficient of } \\
\text { variation }\end{array}$ & 5.84 & Predicted $\mathrm{R}^{2}$ & 0.8004 \\
\hline & & $\begin{array}{l}\text { Adequate } \\
\text { Precision }\end{array}$ & 92.3310 & & & $\begin{array}{l}\text { Adequate } \\
\text { Precision }\end{array}$ & 18.1773 \\
\hline
\end{tabular}

Particle size and zeta potential values indicated F7 to be the best selection. A second-time optimization was done using F7 coated core to achieve further satisfactory extract loading (table 7). Here the amount of extract and time of stirring were varied.
Stirring time was varied between 1 to $6 \mathrm{~h}$, but best results were observed at $2 \mathrm{~h}$. $500 \mathrm{mg}$ of F7 coated core and $1000 \mathrm{mg}$ of extract along with $2 \mathrm{~h}$ of stirring yielded $37.22 \%$ of average extract loading.

Table 7: Formulation optimization-2

\begin{tabular}{lllll}
\hline Formulation code & $\begin{array}{l}\text { Amount of F-7 } \\
\text { coated core } \mathbf{( m g )}\end{array}$ & Amount of extract (mg) & Time of stirring (h) & $\begin{array}{l}\text { Average percentage extract loading } \\
\text { (\%) }\end{array}$ \\
\hline F-A & 500 & 500 & 2 & $14.88 \pm 0.31$ \\
F-A & 500 & 500 & 4 & $10.11 \pm 0.15$ \\
F-B & 500 & 1000 & 2 & $37.22 \pm 0.08$ \\
F-C & 500 & 2 & $15.96 \pm 0.06$ \\
\hline
\end{tabular}

Results represent the average of three independent experiment \pm SD 


\section{FTIR study}

FTIR spectra (fig. 3A) of extract loaded aquasome showed peaks at $567.07 \mathrm{~cm}^{-1}, 601.79 \mathrm{~cm}^{-1}$ and $1037.70 \mathrm{~cm}^{-1}$, which are for phosphate group present in calcium phosphate. FTIR spectra of Chelidonium majus L. extract (fig. 3B) showed a peak at 1080.14 $\mathrm{cm}^{-1}$ due to stretching vibrations $\mathrm{C}-\mathrm{O}$ group of carbohydrate. In fig. $3 \mathrm{~B}$, peak at $1388.75 \mathrm{~cm}^{-1}$ was due to $\mathrm{C}-\mathrm{H}$ bending vibration and peak at $1589.34 \mathrm{~cm}^{-1}$ was due to vibration of $\mathrm{C}=0$ group present in aromatic components $[20,21]$. These peaks were shifted a little in FTIR spectra of aqua some loaded with Chelidonium majus L. (fig. 3A) where peaks were observed at $1419.61 \mathrm{~cm}^{-1}$ and $1604.77 \mathrm{~cm}^{-1}$. FTIR results indicated that Chelidonium majus $L$. extract was successfully loaded onto coated cores and prepared nanoparticles were stable without any interactions between core, carbohydrate and extract.

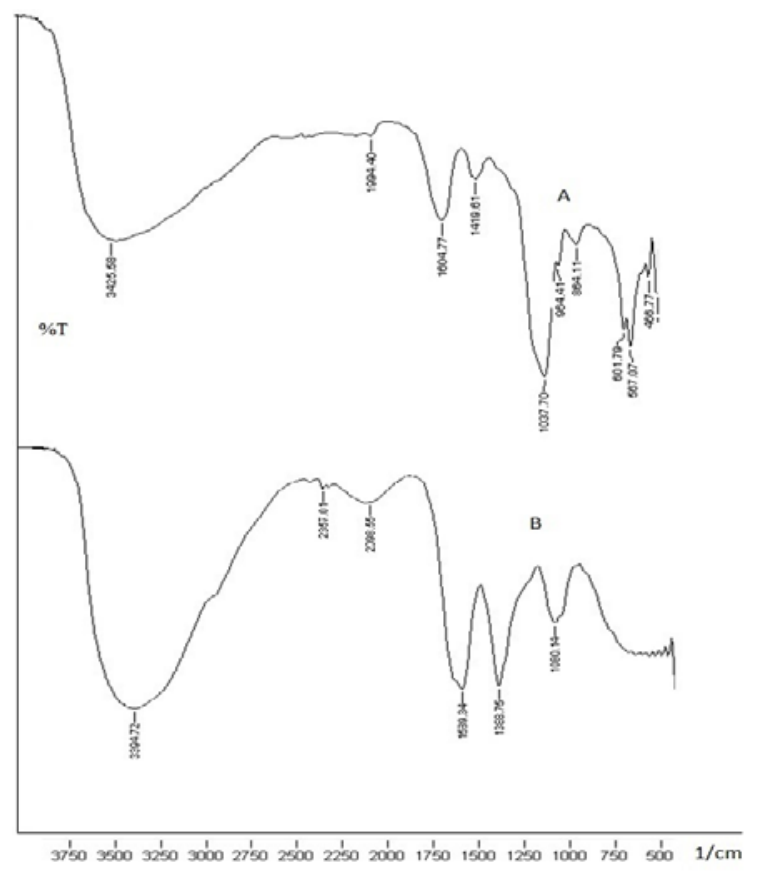

Fig. 3: FTIR spectra of extract loaded aquasome (A) and Chelidonium majus L. extract (B)

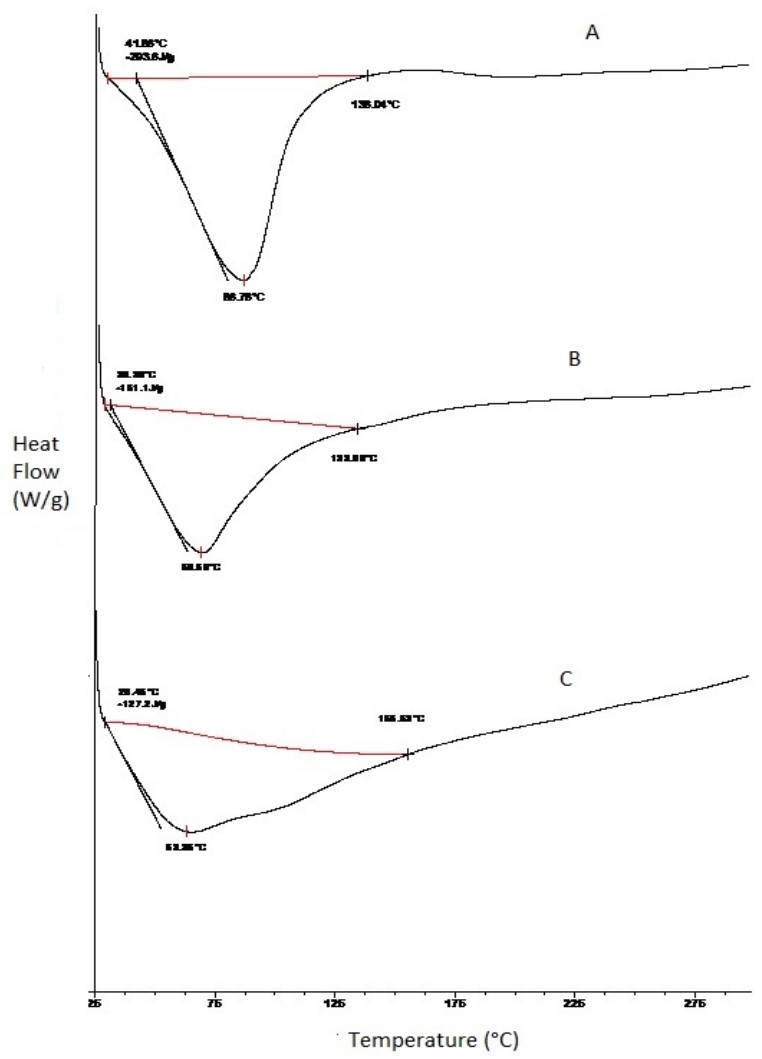

Fig. 4: DSC thermogram of calcium phosphate (A), lactose coated calcium phosphate (B) and extract loaded aquasomes (C) 


\section{Differential scanning calorimetry (DSC)}

Endothermic peak of calcium phosphate showed peak at $86.78{ }^{\circ} \mathrm{C}$ (fig. 4A). Lactose coated core and extract loaded nanoparticles showed endothermic peaks at $68.50^{\circ} \mathrm{C}$ (fig. 4B) and $63.36^{\circ} \mathrm{C}$ (fig. 4C). Shift in peaks observed due to the adsorption of lactose and extract. Endothermic peaks indicated heat absorption during phase transitions. Also, all the peaks indicated the absence of incompatibility between individual components of the formulation.

\section{X-ray diffraction study (XRD)}

XRD graphs of calcium phosphate core, lactose coated core and extract loaded nanoparticles showed intensity lies in between $129-2111 \mathrm{cps}$ (fig. 5A), 76-2307 cps (fig. 5B) and 165-2998 cps (fig. 5C). In XRD graph of extract loaded aquasome (fig. $5 \mathrm{C}$ ), peaks were appeared indicative of amorphous nature of the formulation. Also few sharp peaks were present due to presence of crystalline calcium phosphate. XRD peaks implied that components of the formulation remained stable in the formulation.

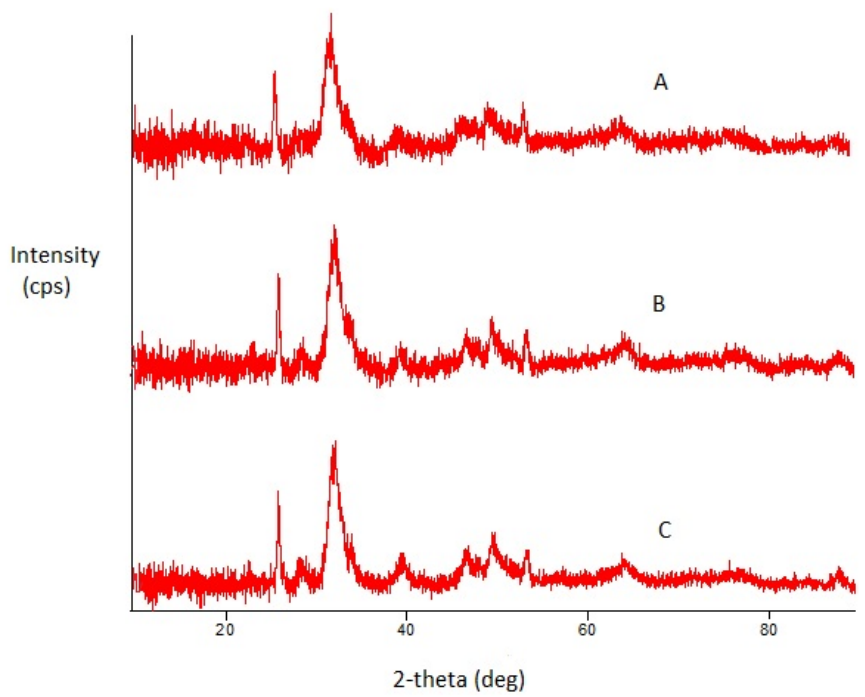

Fig. 5: XRD peaks, A: calcium phosphate, B: lactose coated calcium phosphate, C: extract loaded aquasomes

\section{In vitro dissolution study}

All of the formulations showed initial burst release followed by controlled release during in vitro dissolution study. Release mechanism of all the formulations during dissolution followed
Korsmeyer-peppas and Korsmeyer-peppas with lag model. Release pattern was shown in fig. 6. Since in vitro release pattern followed Korsmeyer-peppas model, it indicated to the fact that extract release from nanoparticles was occurred by diffusion mechanism.
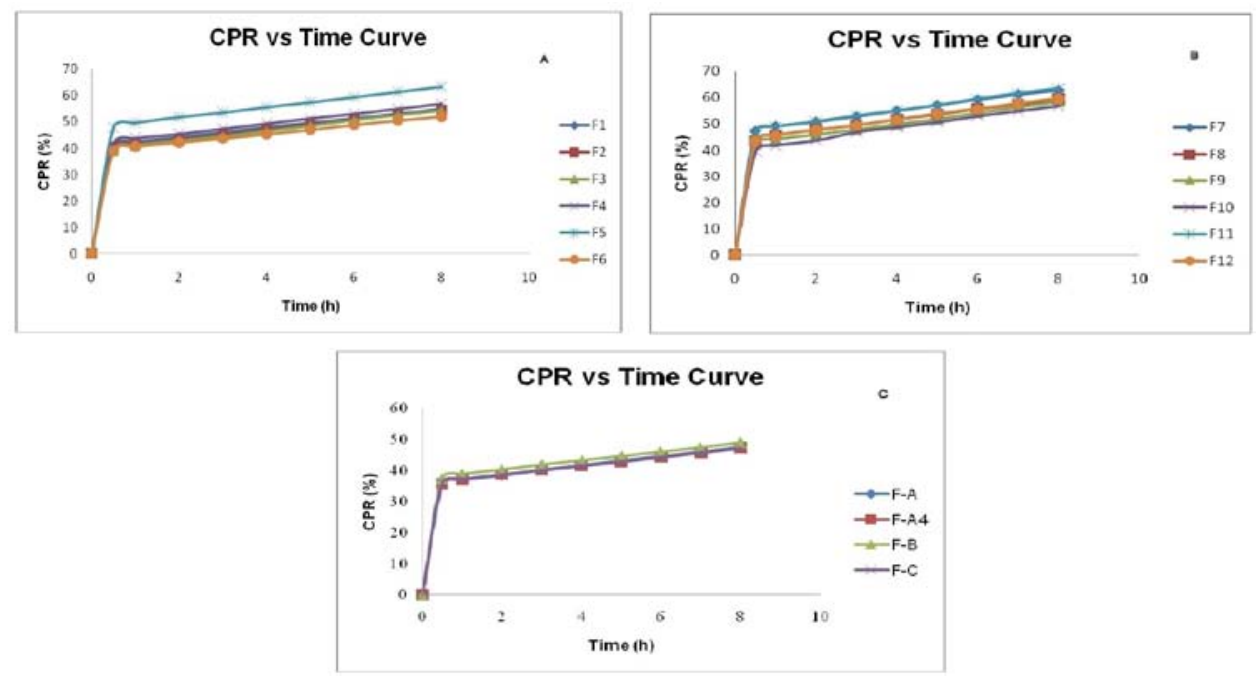

Fig. 6: CPR vs time curve of F1-F6 (A), F7-F12 (B) and F-A to F-C (C) indicating in vitro extract release pattern

\section{Distribution of fluorescence doped nanoparticles in different} organs

Histopathology slides of liver, lung and heart specimens were observed under a fluorescent microscope. Microscopic images showed the distribution of aquasomes in the heart, liver and lung (fig. 7). Presence of large numbers of fluorescent particles was observed in histopathology specimen of the liver (fig. 7B). Very few fluorescent particles were observed in both heart (fig. 7A) and lungs (fig. 7C) specimens. While comparing, heart specimen contained a few higher numbers of particles than lungs. From the observations, it could be stated that these surface-modified extract carriers of calcium phosphate will be an excellent approach for liver targeting. 

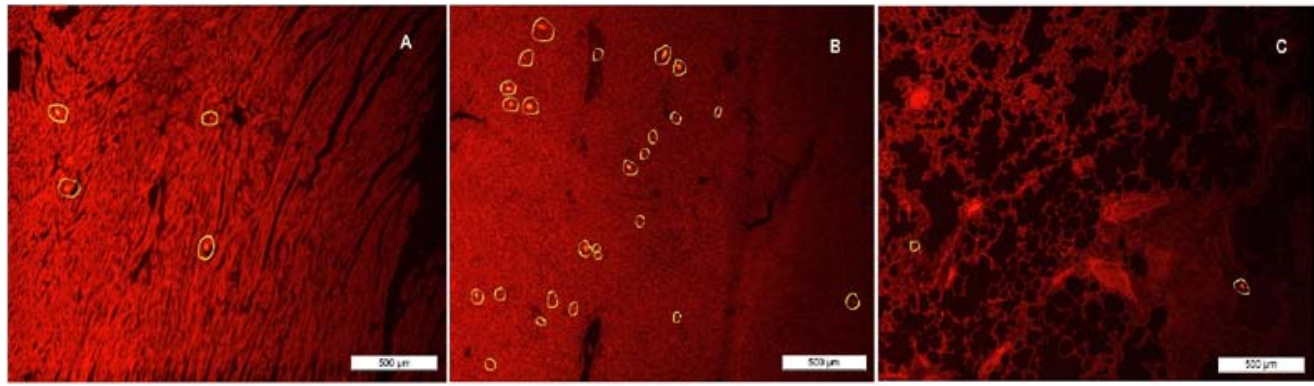

Fig. 7: Distribution of extract loaded aquasomes showed in fluorescence microscopic histopathology images of heart (A), liver (B) and lung (C) of wistar albino rat. Presence of nanoparticles marked with yellow circle

\section{In vivo study}

Histopathology image of normal group (fig. 8A) showed normal cellular and sinusoidal pattern. No necrosis or dilation or congestion was noted in this group. Paracetamol induced hepatotoxic group (fig. 8B) showed cellular necrosis, infiltration of fatty matter and congested sinusoids. Silymarin treated group (fig. 8C) showed normal cellular structure depicting protection against paracetamol-induced toxic effects on hepatocytes. Both CMF (fig. 8D) treated group and CME treated group (fig. 8E) showed the appearance of normal hepatocytes. Sinusoidal congestion was more evidenced in the extract treated group compared to the formulation treated group. It could be concluded that CM extract exerted protective action against paracetamol-induced liver damage similarly like other plant extracts $[22,23]$. Paracetamol caused liver cell damage by covalent binding with DNA [24]. So here it could be said that CM extract might hinder binding property of paracetamol with DNA.

But surface-modified nanoparticles of CM extract showed much better protection due to efficient liver targeting. It was also quite evidenced that both silymarin and CM showed a similar way of protection mechanism during paracetamol-induced liver injury.

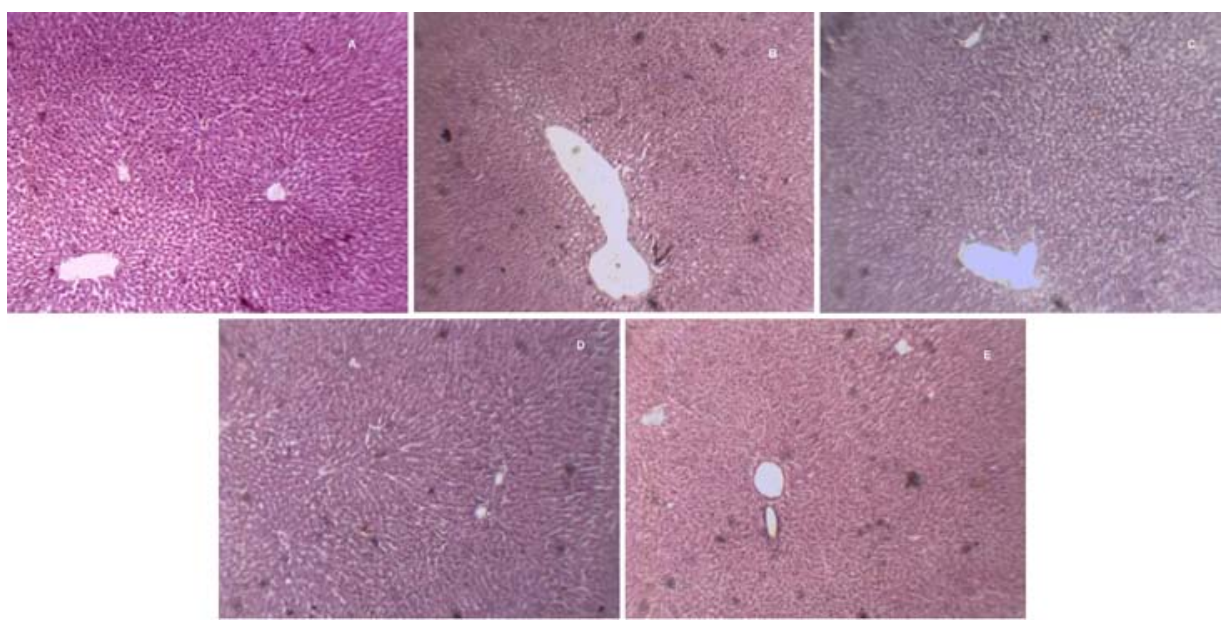

Fig. 8: Histopathological images of liver specimens of wistar albino rats, A: normal treatment group (Gr-N), B: paracetamol treated group (Gr-P1), C: silymarin+paracetamol treated group (Gr-P2), D: CMF+paracetamol treated group (Gr-P3), E: CME+paracetamol treated group (Gr-P4)

Rifampicin-isoniazid induced hepatotoxic group (fig. 9A) showed the presence of degenerative cells, dilated sinusoids, and vacuolization denoting toxic hepatocellular reaction. Silymarin treated group showed effective signs of hepatoprotection with less degenerative cells and vacuolization (fig. 9B). CMF treated group (fig. 9C) showed a better sign of protection than extract-treated group (fig. 9D). Silymarin action of prevention is via reduction of oxidative stress or, more specifically, reduction of free radical production and it was reported to be used as a standard hepatoprotective agent to compare the effect of other plant extracts in rifampicin and isoniazid induced liver damage [25-27]. Here it could be seen that CM acted in a similar manner like silymarin to prevent hepatocytes from oxidative damage.
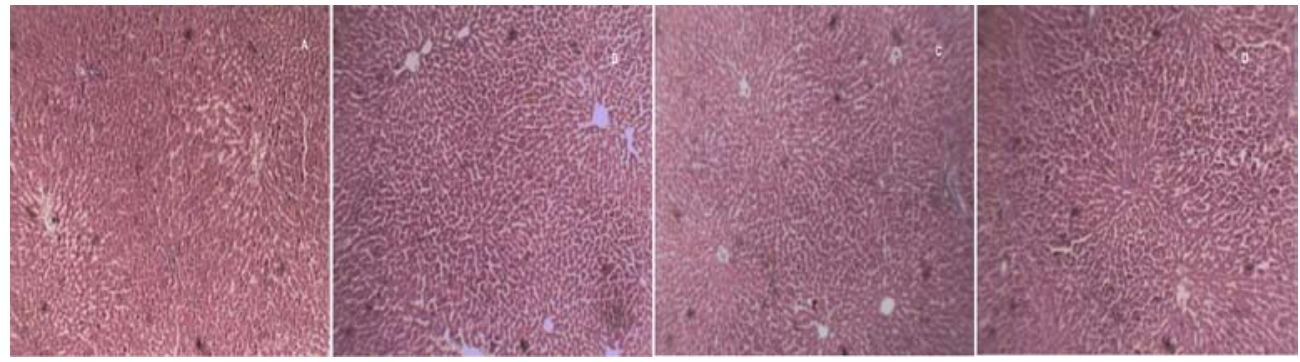

Fig. 9: Histopathological images of liver specimens of wistar albino rats, A: rifampicin+isoniazid treated group (Gr-RI1), B: silymarin+rifampicin+isoniazid treated group (Gr-RI2), C: CMF+rifampicin+isoniazid treated group (Gr-RI3), D: CME+rifampicin+isoniazid treated group (Gr-RI4) 
Here cisplatin group (fig. 10A) showed the presence of degenerative cells, necrosis around central vein along with vacuolization. Silymarin treated group (fig. 10B) showed normal hepatocytes, few vacuolizations and sinusoidal dilations. Both CM treated groups (fig. 10C and fig. 10D) showed normal cellular appearance though better protection was observed in the formulation treated group. Still sinusoids were dilated in some areas of both groups. Prevention of toxic damage of cisplatin by silymarin was reported by Abdelmeguid et al., 2010 [28]. Prevention mechanism included antioxidant activity of silymarin by blocking the production of free radicals in oxidative stress. Here in this study, it could be seen by comparing histopathological images of silymarin, CMF and CM extract-treated group that both CM and silymarin treated group showed similar preventive mechanism in the cellular level. So, it could be said that $\mathrm{CM}$ also generated antioxidant activity or rather say preventive action very much similar like silymarin.

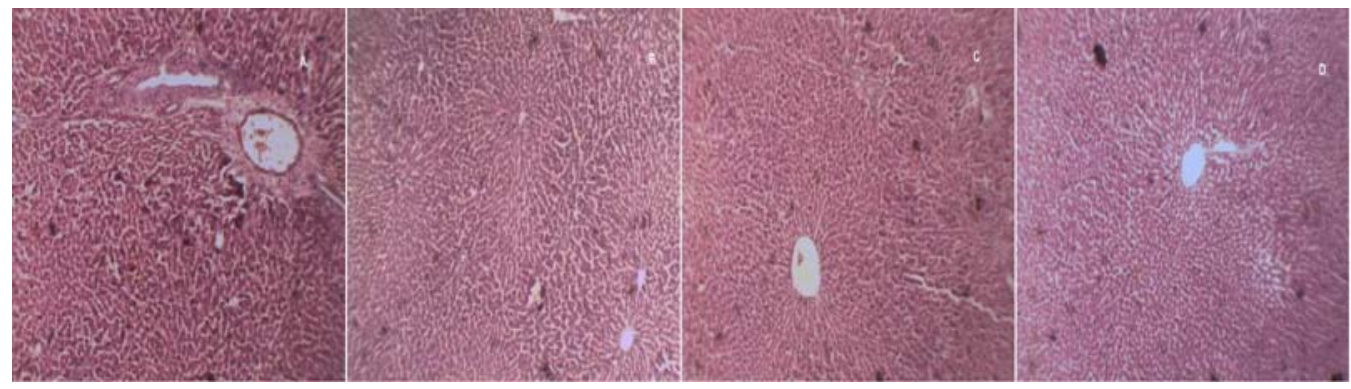

Fig. 10: Histopathological images of liver specimens of wistar albino rats, A: cisplatin treated group (Gr-C1), B: silymarin+cisplatin treated group (Gr-C2), C: CMF+cisplatin treated group (Gr-C3), D: CME+cisplatin treated group (Gr-C4).

Gr-CIN (fig. 11A) showed normal hepatocellular structure, whereas $\mathrm{CCl}_{4}$ induced hepatotoxic group (fig. 11B) showed the appearance of necrosis, dilation of sinusoids and fatty infiltration. In Silymarin treated group (fig. 11C) cells appeared near normal although sinusoidal dilation was visible. Both CMF treated group (fig. 11D) and CME group (fig. 11E) showed fatty infiltration and necrosis. Appearance of fatty droplets during $\mathrm{CM}$ treatment in $\mathrm{CCl}_{4}$ induced hepatotoxicity was similarly visible in the research work of Mitra et al., 1996 [29]. Intensity of sinusoidal congestion was higher in extract-treated group than CMF treated group and by comparing two of the histopathology images it could be concluded that sinusoids were in the recovering stage in the formulation treated group and fatty infiltration was higher in extract-treated group than formulation treated group. It could be concluded that CM extract generated protective action by restoring the cellular regeneration process [30,31]. Extract loaded aquasomes prevented toxic damage to hepatocytes to a level higher than only extract treatment but not in the way of silymarin did.

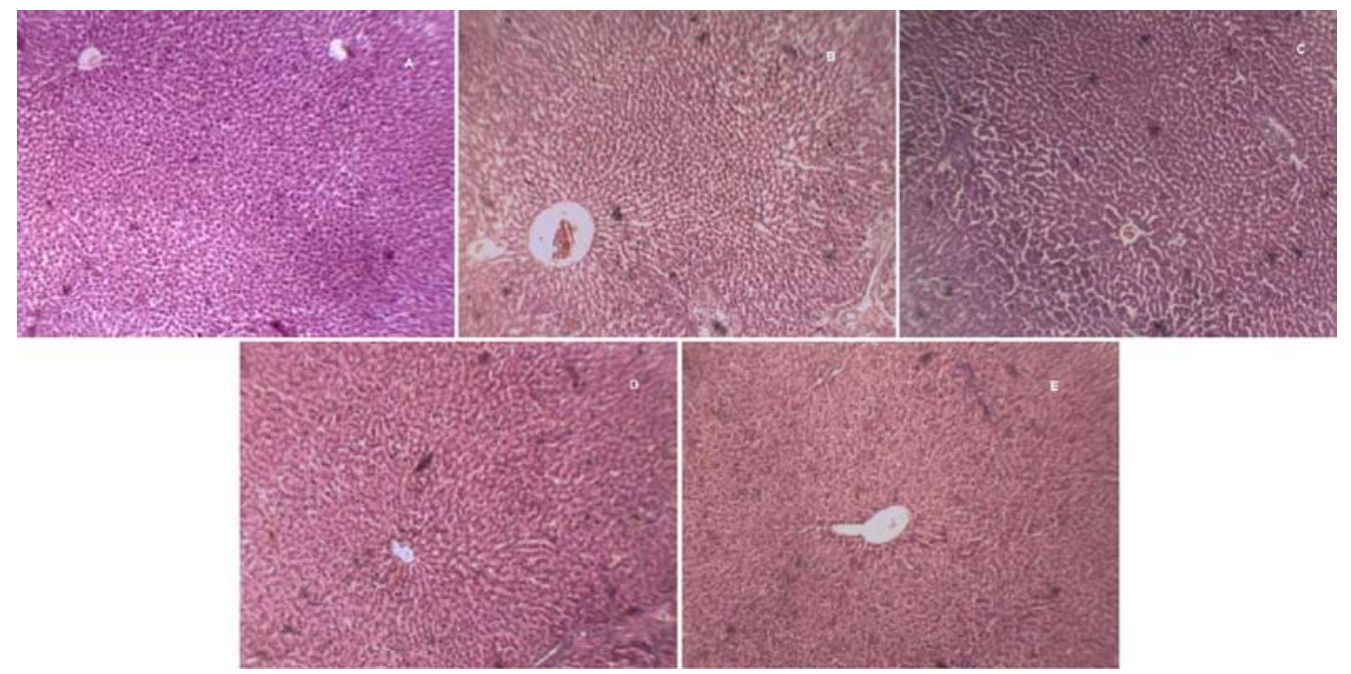

Fig. 11: Histopathological images of liver specimens of wistar albino rats, $\mathrm{A}$ : normal treatment group (only vehicle, $\mathrm{Gr}-\mathrm{ClN}_{\text {), }} \mathrm{B}$ : $\mathrm{CCl}{ }_{4}$ treated group (Gr-Cl1), C: silymarin+CCl${ }_{4}$ treated group (Gr-Cl2), D: $\mathrm{CMF}+\mathrm{CCl}_{4}$ treated group (Gr-Cl3), $\mathrm{E}: \mathrm{CME}+\mathrm{CCl}_{4}$ treated group (Gr-Cl4)

\section{CONCLUSION}

In this work, surface modification of calcium phosphate nanoparticles with lactose resulted in the successfully loading of Chelidonium majus L. extract. Extract adsorption via non-covalent interactions was further confirmed by FTIR interpretations. Glassy, aqueous like environment provided by carbohydrate, created a potential sustaining and preserving effect to the plant extract. Extract loading and particle size, both of the responses were proved to be significant and termed as good signal during statistical optimization. All the formulations exhibited satisfactory extract loading and prepared nanoparticles were stable by conforming to characterization study, particle size and zeta potential results. Also, it was evidenced that aquasomes of Chelidonium majus $L$. extract were likely to end up more in liver than in the heart and lungs. Chelidonium majus L. extract loaded nanoparticle showed efficient cellular protective action in liver in a similar manner like silymarin. It is known that plant extract contain many hydrophobic components. Also, these surface-modified nanocrystalline materials are best choice for delivering typically water-insoluble components. So, it can be deduced that surface retailoring of calcium phosphate nanoparticles with polyhydroxy oligomers will make it as a lucrative choice for an excellent extract carrier with liver targeting proficiency in the future. 


\section{ABBREVIATION}

Chelidonium majus L., CM, Alginate coated Chelidonium majus L. extract loaded nanoparticle, CMF, Chelidonium majus L. extract, CME, paracetamol, P, Rifampicin-isoniazid, RI, Cisplatin, C, Carbon tetrachloride, $\mathrm{CCl}_{4} / \mathrm{Cl}$, Group, $\mathrm{Gr}$

\section{ACKNOWLEDGMENT}

This work was supported by fellowships from DST-Inspire, Department of Science and Technology, Ministry of Science and Technology, Government of India (DST/INSPIRE Fellowship/ 2015/IF150359).

\section{FUNDING}

Nil

\section{AUTHORS CONTRIBUTIONS}

Author Sritoma Banerjee carried out all the works and co-author Kalyan Kumar Sen guided her throughout the whole work.

\section{CONFLICT OF INTERESTS}

The authors declare no conflict of interest with other organizations or individuals regarding the content or publication of this paper.

\section{REFERENCES}

1. Kossovsky N, Gelman A, Sponsler EE, Hnatyszyn HJ, Rajguru S, Torres M, et al. Surface-modified nanocrystalline ceramics for drug delivery applications. Biomaterials 1994;15:1201-7.

2. Khopade AJ, Khopade S, Jain NK. Development of haemoglobin aquasomes from spherical hydroxyapatite cores precipitated in the presence of poly(amidoamine) dendrimer. Int J Pharm (Amsterdam, Neth) 2002;241:145-54.

3. Patil S, Pancholi SS, Agrawal S, Agrawal GP. Surface-modified mesoporous ceramics as a delivery vehicle for haemoglobin. Drug Delivery 2004;11:193-9.

4. Cherian AK, Rana AC, Jain SK. Self-assembled carbohydratestabilized ceramic nanoparticles for the parenteral delivery of insulin. Drug Dev Ind Pharm 2000;26:459-69.

5. Kossovsky N, Gelman A, Hnatyszyn HJ, Rajguru S, Garrell RL, Torbati S, et al. Surface-modified diamond nanoparticles as antigen delivery vehicles. Bioconjugate Chem 1995;6:507-11.

6. Kossovsky N, Gelman A, Sponsler E, Rajguru S, Torres M, Mena $\mathrm{E}$, et al. Preservation of surface-dependent properties of viral antigens following immobilization on particulate ceramic delivery vehicles. J Biomed Mater Res 1995;29:561-73.

7. Goyal AK, Khatri K, Mishra N, Mehta A, Vaidya B, Tiwari S, et al. Aquasomes-a nanoparticulate approach for the delivery of antigen. Drug Dev Ind Pharm 2008;34:1297-305.

8. Kommineni S, Ahmad S, Vengala P, Subrahmanyam CVS. Sugar coated ceramic nanocarriers for the oral delivery of hydrophobic drugs: formulation, optimization and evaluation. Drug Dev Ind Pharm 2012;38:577-86.

9. Vengala P, Aslam S, Subrahmanyam CVS. Development and in vitro evaluation of ceramic nanoparticles of piroxicam. Latt Am J Pharm 2013;32:1124-30.

10. Vengala P, Dintakurthi S, Subrahmanyam CVS. Lactose coated ceramic nanoparticles for oral drug delivery. J Pharm Res 2013;7:540-5.

11. Rawat M, Singh D, Saraf S, Saraf S. Development and in vitro evaluation of alginate gel-encapsulated, chitosan-coated ceramic nanocores for oral delivery of enzyme. Drug Dev Ind Pharm 2008;34:181-8.

12. Goyal AK, Rawat A, Mahor S, Gupta PN, Khatri K, Vyas SP. Nanodecoy system: a novel approach to design hepatitis B vaccine for immunopotentiation. Int J Pharm (Amsterdam, Neth) 2006;309:227-33.
13. Kaur K, Kush P, Pandey RS, Madan J, Jain UK, Katare OP. Stealth lipid-coated aquasomes bearing recombinant human interferon- $\alpha$ $2 \mathrm{~b}$ offered prolonged release and enhanced cytotoxicity in ovarian cancer cells. Biomed Pharmacother 2015;69:267-76.

14. Gilca M, Gaman L, Panait E. Chelidonium majus-an integrative review: traditional knowledge versus modern findings. Forschende Komplementärmedizin 2010;17:241-8.

15. Zielinska S, Jezierska Domaradzka A, Wójciak Kosior M. Greater celandine's ups and downs-21 centuries of medicinal uses of Chelidonium majus from the viewpoint of today's pharmacology. Front Pharmacol 2018;9:1-29.

16. Banerjee S, Sen KK. Qualitative and quantitative evaluation study along with method development and validation for UV spectrophotometric analysis of Chelidonium majus L. extract. J Pharmacogn Phytochem 2019;8:4629-36.

17. Prabu P, Chaudhari AA, Dharmaraj N, Khil MS, Park SY, Kim HY. Preparation, characterization, in vitro drug release and cellular uptake of poly(caprolactone) grafted dextran copolymeric nanoparticles loaded with an anticancer drug. J Biomed Mater Res, Part A 2008;90:1128-36.

18. Kumar R, Nagarwal RC, Dhanawat M, Pandit JK. In vitro and in vivo study of indomethacin loaded gelatin nanoparticles. J Biomed Nanotechnol 2011;7:1-9.

19. Anuradha, Joshi JC, Gulati K, Ray A, Roy I. Fluorophore-doped calcium phosphate nanoparticles for non-toxic biomedical applications. RSC Adv 2014;4:40449-55.

20. Zavoi S, Fetea F, Ranga F, M Pop R, Baciu A, Socaciu C. Comparative fingerprint and extraction yield of medicinal plant phenolics with hepatoprotective potential, as determined by UV-vis and FT-MIR spectroscopy. Not Bot Horti Agrobot ClujNapoca 2011;39:82-9.

21. Dobrucka R, Dlugaszewska J, Kaczmarek M. Cytotoxic and antimicrobial effects of biosynthesized $\mathrm{ZnO}$ nanoparticles using of Chelidonium majus extract. Biomed Microdevices 2017;20:5.

22. Srividya G, Adilaxmamma K, Srilatha CH. Protective effect of Acorus calamus rhizome in paracetamol exposure induced hepatotoxicity in rats: biochemical and histopathological study. Int J Curr Pharm Res 2018;10:7-10.

23. Sitorus P, Nerdy N. Hepatoprotective activity of Vernonia amygdalina leaf ethanolic extract in white rats induced by paracetamol. Asian J Pharm Clin Res 2018;11:562-4.

24. Garcia Nino WR, Zazueta C. Ellagic acid: pharmacological activities and molecular mechanisms involved in liver protection. Pharmacol Res 2015;97:84-103.

25. Bais B, Saiju P. Ameliorative effect of Leucas cephalotes extract on isoniazid and rifampicin induced hepatotoxicity. Asian Pac J Trop Biomed 2014;4(Suppl 2):S633-8.

26. Eminzade S, Uraz F, Izzettin FV. Silymarin protects the liver against toxic effects of anti-tuberculosis drugs in experimental animals. Nutr Metab (Lond) 2008;5:1-8.

27. Hussain T, Gupta RK, Sweety K. Evaluation of the antihepatotoxic potential of Solanum xanthocarpum fruit extract against antitubercular drugs induced hepatopathy in experimental rodents. Asian Pac J Trop Biomed 2012;2:454-60.

28. Abdelmeguid NE, Chmaisse HN, Zeinab NSA. Silymarin ameliorates cisplatin-induced hepatotoxicity in rats: histopathological and ultrastructural studies. Pak J Biol Sci 2010;13:463-79.

29. Mitra S, Sur RK (deceased), Roy A, Mukherjee AS. Effect of Chelidonium majus L. on experimental hepatic tissue injury. Phytother Res 1996;10:354-6.

30. Rajalingam D, Varadharajan R, Palani S. Evaluation of hepatoprotective and antioxidant effect of Combretum albidum G. Don against ccl4 induced hepatotoxicity in rats. Int J Pharm Pharm Sci 2016;8:218-23.

31. Mushtaq A, Masoodi MH, Wali AF, Ganai BA. Multiple treatments of eremurus himalaicus extracts ameliorates carbon tetrachlorideinduced liver injury in rats. Int J Pharm Pharm Sci 2016;8:24-7. 